\author{
ANNALES \\ POLONICI MATHEMATICI \\ XLI (1983)
}

\title{
On extremal solutions to infinite dimensional non-linear second order systems
}

\author{
by Russell C. Thompson (Logan, Utah)
}

\begin{abstract}
Consider the following boundary value problem for an infinite system of second order ordinary differential equations:

$$
\begin{gathered}
-D^{2} y+f(x, y, D y)=0, \quad x \in(0,1), \\
g(y(0), D y(0))=0=h(y(1), D y(1)) .
\end{gathered}
$$

In the above problem $y, f, g, h$ have values in the set of real sequences indexed by a subset $J$ of the set of integers and $D^{2} y, D y$ denote, respectively, certain weak second and first derivatives of $y$. In this paper some results are obtained on the existence and the monotone approximation of maximal and minimal solutions to (I) using diflerential inequality techniques. An example is presented at the conclusion of the paper in which the results are used to obtain convergence of the method of lines for elliptic boundary value problems in an unbounded domain.

Key words. Second order differential equations, infinite systems, boundary value problems, maximal-minimal solutions, differential inequalities, elliptic boundary value problems, method of lines.
\end{abstract}

1. Introduction. In this paper, we investigate the existence and monotone approximation of maximal and minimal solutions to boundary value problems for systems of second order ordinary differential equations of the form

$$
\begin{gathered}
-D^{2} y+f(x, y, D y)=0, \quad x \in(0,1), \\
g(y(0), D y(0))=0=h(y(1), D y(1)) .
\end{gathered}
$$

In the above problems $y, f, g$, and $\boldsymbol{h}$, have values in the set of real sequences indexed by a set $J \subseteq Z$, where $Z$ is the set of integers. $D y$ and $D^{2} y$ denote certain weak first and second derivatives, respectively, of $y(x)$. This system is infinite dimensional or finite dimensional as the index set is an infinite or finite subset of $Z$, respectively.

In some earlier work on solutions to boundary value problems of this type in the infinite dimensional case, the problem has been formulated in one of the Banach spaces $l^{p}, 1 \leqslant p \leqslant \infty$ (see for example, reference [14]). These results have the disadvantages, from the point of view of certain applications, that the derivative is defined in terms of the norm-topology in the Banach 
space (implying a type of uniform differentiability with respect to the index $i$ ); and second that, in order to apply fixed point theory to show the existence of solutions, some sort of compactness condition in the form of a rather restrictive growth condition is required on the functions $f(x, y, z)$, $\boldsymbol{g}(\boldsymbol{y}, z)$, and $\boldsymbol{h}(\boldsymbol{y}, z)$. One such application which produces problems of the form (1.1), (1.2) is the approximate solution of elliptic partial differential equations, using the method of lines. In some recent work on this problem (see reference [11]), we have shown that, for problems arising in the method of lines, the formulation in the Banach space $l^{p}$ can be avoided by taking advantage of the fact that the coupling in (1.1), (1.2) arises from numerical differentiation and is hence local in nature $(f(x, y, z), g(y, z)$, and $h(y, z)$ are row finite). The existence results in [11] require essentially no growth condition beyond this row finiteness; however, in order to obtain uniqueness of solutions, some growth conditions seem to be needed.

In the last two years a number of results have begun to appear on the existence of extremal solutions for systems, including some recent results for infinite systems (see e.g. [1], [2], [3]). In these results the extremal solutions are obtained iteratively, starting with solutions of certain differential inequalities. A similar technique, sometimes referred to as Chaplygins method has been used for some time in investigations of monotone methods for initial value problems for systems of ordinary differential equations and parabolic boundary value problems (see references [7], [8]).

In the present work, we show that if $f$ satisfies a Kamke condition and a growth condition in $D \boldsymbol{y}$, if $\boldsymbol{g}$ and $\boldsymbol{h}$ satisfy a monotonicity condition in $\boldsymbol{y}$ and $D y$, and if certain differential inequalities have solutions, then there exist monotone sequences of approximate solutions which converge to the extremal solutions to problem (1.1), (1.2). Our approach was suggested by some results by Mlak and Olech [8] for initial value problems, however, the techniques are similar to those used in the results on boundary value problems mentioned above.

In Section 2 we present an extension of a minimum principle, appearing in reference [12], to the case of weak derivatives and nonlinear boundary conditions. This result gives sufficient conditions for the uniqueness of solutions to (1.1), (1.2) and gives a comparison principle for solutions to differential inequalities. The next two sections, Sections 3 and 4 , contain snme preliminary notation and lemmas used in subsequent sections to construct the approximate solutions. Section 5 contains the main results on the existence of extremal solution to (1.1), (1.2). Given results on existence of maximal and minimal solutions to one dimensional problems our construction of approximate solutions is an iterative one.

In Section 6, after strengthening the hypotheses on (1.1), (1.2) to guarantee uniqueness of solutions, we give a construction of approximate solutions which suggests a method of local error estimation in the solution of 
truncated systems. These results are illustrated on a problem arising in the method of lines solution of an elliptic boundary value problem in Section 7.

2. A minimum principle. In this section, we present a minimum principle and a comparison theorem for systems of differential inequalities corresponding to (1.1), (1.2). These results are similar to Theorem 2.1 and Theorem 3.1 of reference [12] differing in that the derivatives here are weak derivatives and the boundary conditions may be non-linear. The following are some properties of ordered Banach spaces which are used in the section.

Let $B$ be a Banach space with norm $|\cdot|$. Denote the set of continuous linear functionals by $B^{*}$ and the unit ball of $B^{*}$ by $U^{*}$. If $K^{*} \subseteq U^{*}$, then $\bar{K}^{*}$ denotes the closure of $K^{*}$ in the weak $*$ topology. A closed subset $K \subseteq B$ is called a cone if: (i) $\alpha K \subseteq K$ for every $\alpha \geqslant 0$; (ii) $K$ is convex; and (iii) $K \cap(-K)=\{0\}$. An element $\varphi \in B^{*}$ is called a positive linear functional with respect to $K$ if $\varphi(u) \geqslant 0$ for every $u \in K$. The set $K^{*} \subseteq B^{*}$ is said to generate the cone $K \subseteq B$ if $K$ is the intersection of the half-spaces $K=\bigcap\{\boldsymbol{u} \in \boldsymbol{B}: \varphi(\boldsymbol{u})$ $\left.\geqslant 0, \varphi \in K^{*}\right\}$. A cone $K$ is said to be solid if int $K$ is non-empty. If $u_{0} \in$ int $K$ and $\varphi$ is a positive linear functional, not identically zero, then $\varphi\left(\boldsymbol{u}_{0}\right)>0$. If $K$ is a cone and a partial ordering is defined by setting $u \leqslant v$ if and only if $\boldsymbol{u}-\boldsymbol{v} \in K$ and $\boldsymbol{u}<\boldsymbol{v}$ if and only if $\boldsymbol{u}-\boldsymbol{v} \in$ int $K$, then $\leqslant$ and $<$ have the usual properties of the sign of inequality. Let $K^{*} \subseteq U^{*}$ be such that $K^{*}$ generates a solid cone $K$ and suppose there exists a $u_{0} \in$ int $K$ and a $\delta>0$ such that $\inf \left\{\varphi\left(u_{0}\right): \varphi \in K^{*}\right\} \geqslant \delta$. Define the set of functionals $K_{u_{0}}^{*}$ by means of the equation

$$
K_{u_{0}}^{*}=\left\{\varphi \in B^{*} \mid \varphi(\boldsymbol{u})=\frac{\delta}{\psi\left(\boldsymbol{u}_{0}\right)} \psi(\boldsymbol{u}), \psi \in K^{*}, \boldsymbol{u} \in B\right\} .
$$

This set has the properties: $K_{u_{0}}^{*} \subseteq U^{*}, K_{u_{0}}^{*}$ generates $K$; and $\varphi\left(u_{0}\right)=\delta$ for every $\varphi \in K_{u_{0}}^{*}$.

A subspace $\Gamma \subseteq B^{*}$ is said to be a total space of functionals if $\varphi(\boldsymbol{u})=0$ for every $\varphi \in \Gamma$ implies $\boldsymbol{u}=\mathbf{0}$ (see [5], p. 418). Let $\Gamma$ be a total space of functionals on $B$ and let $u:[0,1] \rightarrow B$. Du and $D^{2} u$ are called weak first and second derivatives of $\boldsymbol{u}$ with respect to $x$ in the $\Gamma$-topology if the following equations hold for all $\varphi \in \Gamma$ :

$$
\begin{gathered}
\lim _{h \rightarrow 0} h^{-2} \varphi\left([u(x+h)-2 u(x)+u(x-h)]-h^{2} D^{2} u(x)\right)=0, \\
\lim _{h \rightarrow 0} h^{-1} \varphi([u(x+h)-u(x)]-h D u(x))=0 .
\end{gathered}
$$

It follows from these equations that $\varphi(D u(x))=\frac{d}{d x} \varphi(\boldsymbol{u}(x))$ and $\varphi\left(D^{2} \boldsymbol{u}(x)\right)$ $=\frac{d^{2}}{d x^{2}} \varphi(u(x))$.

Suppose $K^{*} \subseteq B^{*}$ generates a cone $K \subseteq B$. Let $\Gamma=\operatorname{span}\left(K^{*}\right)$, then $\Gamma$ is a total set of functionals on $B$ because of (iii). 
The set $C([0,1], B)$ denotes the set of (strongly) continuous functions from $[0,1]$ into $B$ and $C \Gamma^{2}([0,1], B)$ the set of functions $u:[0,1] \rightarrow B$ such that $\varphi(u(x)) \in C^{2}[0,1]$ for each $\varphi \in \Gamma$.

THEOREM 2.1. Let $K$ be a solid cone in $B$ generated by a subset $K^{*} \subseteq U^{*}$ let $\boldsymbol{u}_{0} \in$ int $K$ be such that inf $\left\{\varphi\left(u_{0}\right)\right\} \geqslant \delta>0$ for some $\delta>0$. Let $\boldsymbol{F}[0,1] \times$ $\times B \times B \rightarrow B, G: B \times B \rightarrow B$ and $\boldsymbol{H}: B \times B \rightarrow B$ satisfy the following conditions:

(i) for every $(x, u, w),(x, v, z) \in[0,1] \times B \times B$,

$$
\begin{gathered}
\varphi \in \bar{K}_{u_{0}}^{*}, \varphi(\boldsymbol{u}-\boldsymbol{v})=\inf \left\{\varphi(\boldsymbol{u}-\boldsymbol{v}): \varphi \in K_{u_{0}}^{*}\right\} \leqslant 0, \\
\varphi(\boldsymbol{w}-\boldsymbol{z})=0 \Rightarrow \varphi(\boldsymbol{F}(x, \boldsymbol{u}, \boldsymbol{w})) \leqslant \varphi(\boldsymbol{F}(x, \boldsymbol{v}, \boldsymbol{z}))
\end{gathered}
$$

and

$$
\begin{gathered}
\varphi \in \bar{K}_{u_{0}}^{*}, \quad \varphi(\boldsymbol{u}-\boldsymbol{v})=\inf \left\{\varphi(\boldsymbol{u}-\boldsymbol{v}): \varphi \in K_{u_{0}}^{*}\right\}<0, \\
\varphi(\boldsymbol{w}-\boldsymbol{z})=0 \Rightarrow\left\{\begin{array}{l}
\varphi(\boldsymbol{G}(\boldsymbol{u}, \boldsymbol{w}))>\varphi(\boldsymbol{G}(\boldsymbol{v}, \boldsymbol{z})), \\
\varphi(\boldsymbol{H}(\boldsymbol{u}, \boldsymbol{w}))<\varphi(\boldsymbol{H}(\boldsymbol{v}, \boldsymbol{z})) ;
\end{array}\right.
\end{gathered}
$$

(ii) $F(x, 0,0) \leqslant 0, G(0,0) \geqslant 0$, and $H(0,0) \leqslant 0$;

(iii) $F(x, u, w)$ satisfies a Lipschitz condition with respect to $w$ on closed and bounded subsets, of $[0,1] \times B \times B$; and

(iv) $\varphi(\boldsymbol{w}-\boldsymbol{z}) \geqslant 0 \Rightarrow\left\{\begin{array}{l}\varphi(\boldsymbol{G}(\boldsymbol{u}, \boldsymbol{w})) \geqslant \varphi(\boldsymbol{G}(\boldsymbol{u}, \boldsymbol{z})) \\ \varphi(\boldsymbol{H}(\boldsymbol{u}, \boldsymbol{w})) \geqslant \varphi(\boldsymbol{H}(\boldsymbol{u}, \boldsymbol{z})) .\end{array}\right.$

If $u \in C([0,1], B)$ satisfies

$$
\begin{gathered}
-D^{2} \boldsymbol{u}(x)+\boldsymbol{F}(x, \boldsymbol{u}(x), D \boldsymbol{u}(x)) \geqslant 0, \quad x \in(0,1), \\
G(\boldsymbol{u}(0), D \boldsymbol{u}(0)) \leqslant 0 \leqslant \boldsymbol{H}(\boldsymbol{u}(1), D \boldsymbol{u}(1)),
\end{gathered}
$$

then $\boldsymbol{u}(x) \geqslant 0$ for all $x \in[0,1]$.

Proof. Consider first the case in which the expression in (2.1) satisfies $\varphi\left(-D^{2} \boldsymbol{u}(x)+\boldsymbol{F}(x, \boldsymbol{u}(x), D \boldsymbol{u}(x))\right)>0$ for every $\varphi \in K_{u_{0}}^{*}$. Let us assume contrary to the conclusion of the theorem that $u(x) \geqslant 0$ fails for some $x \in[0,1]$. Define a continuous function $\Phi:[0,1] \rightarrow R$ by setting

$$
\Phi(x)=\inf \left\{\varphi(u(x)): \varphi \in K_{u_{0}}^{*}\right\}
$$

(note: the continuity of $\Phi$ follows from that of $u$ ). The assumption that $u(x) \geqslant 0$ does not hold for some $x \in[0,1]$ implies that $\Phi(x)<0$ for some $x \in[0,1]$. By continuity there exists an $x_{0} \in[0,1]$ such that $\Phi\left(x_{0}\right)$ $=\min \{\Phi(x): x \in[0,1]\}$.

Suppose $x_{0}=0$. By Lemma 2.3 of reference [12] there exists a $\psi \in \bar{K}_{u_{0}}^{*}$ such that $\psi(\boldsymbol{u}(0))=\Phi(0)=\min \{\Phi(x): \quad x \in[0,1]\}<0$. Define $y(x)=\psi(\boldsymbol{u}(x))$. Since $y(0) \leqslant y(x)$ for every $x \in[0,1]$, it follows that $0 \leqslant y^{\prime}(0)=\left.\frac{d}{d t}(\psi(u(x)))\right|_{x=0}=\psi(D u(0))$. We obtain from (2.2) and hypothe- 
ses (i)-(ii) that

$$
0 \leqslant \psi(\boldsymbol{G}(\boldsymbol{0}, 0))<\psi(\boldsymbol{G}(\boldsymbol{u}(0), 0)) \leqslant \psi(\boldsymbol{G}(\boldsymbol{u}(0), D \boldsymbol{u}(0))) \leqslant 0 .
$$

It follows from this contradiction that $x_{0}=0$ is not possible. A similar argument shows that $x_{0} \neq 1$ and therefore $x_{0} \in(0,1)$. The argument that this is also impossible now follows from the proof of Theorem 2.1 in [12].

In the general case where (2.1) holds, suppose again that the inequality $\boldsymbol{u}(x) \geqslant 0$ fails at some $x \in I$. Let $\Phi(x)$ and $x_{0}$ be as above and let $\varepsilon>0$ be a number such that $\Phi\left(x_{0}\right)=-4 \varepsilon$. Let $\varrho(x)$ be a solution to the scalar equation $\varrho^{\prime \prime}=(L+1) \varrho^{\prime}$ satisfying $0 \leqslant \varrho<\varepsilon /\left|u_{0}\right|$ and $-1 \leqslant \varrho^{\prime} \leqslant-\gamma<0$ for some $0<\gamma<1$, where $L>0$. Define $w(x)=u(x)+\varrho(x) u_{0}$. Then, making use of (i) and (iv) we have

$$
\boldsymbol{G}(\boldsymbol{w}(0), D w(0))=\boldsymbol{G}\left(\boldsymbol{u}(0)+\varrho(0) u_{0}, D u(0)+\varrho^{\prime}(0) u_{0}\right) \leqslant G(u(0), D u(0)) \leqslant 0,
$$

and, in a similar way,

$$
\boldsymbol{H}(\boldsymbol{w}(0), D \boldsymbol{w}(0)) \geqslant 0 .
$$

Furthermore, if $L$ is the Lipschitz constant for $F(x, v, z)$ corresponding to the set $\left\{(x, y, z) \in[0,1] \times B \times B: x \in[0,1],|y| \leqslant\|u\|+\varepsilon /\left|u_{0}\right|,|z| \leqslant\|D u\|+1\right\}$, then $w(x)$ satisfies the condition, in the first part of the proof, with respect to inequality (2.1). It follows that $w(x) \geqslant 0$ on $[0,1]$. However, at the point $x_{0}$ we have

$$
\inf \left\{\varphi\left(w\left(x_{0}\right)\right): \varphi \in K_{\nu_{0}}^{*}\right\} \leqslant \Phi\left(x_{0}\right)+\inf \left\{\varphi\left(\varrho\left(x_{0}\right) u_{0}\right): \varphi \in K_{u_{0}}^{*}\right\} \leqslant-3 \varepsilon<0
$$

which contradicts the inequality $w(x) \geqslant 0$. The conclusion follows from this contradiction.

THEOREM 2.2. Let $\boldsymbol{f}(x, \boldsymbol{u}, \boldsymbol{w}), \boldsymbol{g}(\boldsymbol{u}, \boldsymbol{w})$, and $\boldsymbol{h}(\boldsymbol{u}, \boldsymbol{w})$ satisfy conditions (i), (iii), and (iv) for $\boldsymbol{F}, \boldsymbol{G}$, and $\boldsymbol{H}$, respectively, in Theorem 2.1. If $\boldsymbol{w}, \boldsymbol{v}, C([0,1], B)$ satisfy

$$
\begin{aligned}
& -D^{2} w(x)+f(x, w(x), D w(x)) \leqslant-D^{2} v(x)+f(x, v(x), D v(x)), \\
& g(w(0), D w(0)) \geqslant g(v(0), D v(0)), \quad h(w(1), D w(1)) \leqslant h(v(1), D v(1)),
\end{aligned}
$$

then $w(x) \leqslant v(x)$ for all $x \in[0,1]$.

Proof. Define functions $F(x, u, D u), G(u, D u)$, and $H(u, D u)$ as follows:

$$
\begin{gathered}
F(x, u, D u) \\
=-D^{2} w(x)+D^{2} u(x)+f(x, w(x), D w(x))+f(x, v(x)-u, D v(x)-D u), \\
G(u, D u)=g(w(0), D w(0))-g(v(0)-u, D v(0)-D u), \\
H(u, D u)=h(w(1), D w(1))-h(v(1)-u, D v(1)-D u) .
\end{gathered}
$$

One easily verifies that the $\boldsymbol{F}, \boldsymbol{G}$, and $\boldsymbol{H}$ defined above satisfy the hypotheses of Theorem 2.1. The conclusion follows by defining $u(x)=v(x)-w(x)$ and applying Theorem 2.1 . 
3. Two lemmas. In this section we present two lemmas which we will refer to repeatedly in the construction of solutions to problem (1.1), (1.2).

The first of these results in a growth condition in $D y$ which is used to obtain bounds on the derivative of certain bounded solutions. In particular, let $B$ be a Banach space, $f:[0,1] \times B \times B \rightarrow B$, and $|\cdot|$ represent the norm in $B$. For $y:[0,1] \rightarrow B$ let $\|y\|=\max _{0 \leqslant x \leqslant 1}|y(x)|$. We will say that $f$ satisfies a Nagumo condition if for every bounded set $Y \subseteq B$, there exists a non-decreasing continuous function $\psi[0, \infty) \rightarrow(0, \infty)$ such that

$$
\lim _{s \rightarrow \infty} \frac{s^{2}}{\psi(s)}=+\infty
$$

and

$$
|f(x, y, z)| \leqslant \psi(|z|), \quad x \in[0,1], y \in Y .
$$

The bounds on the derivatives of solutions are obtained from the following lemma.

LemMa 3.1 (see [10]). Let $\psi(s)$ be a positive, non-decreasing, continuous, real valued function satisfying (3.1) and let $R$ be a positive number. Then there exists a positive constant $M=M(\psi, R)$ such that, if $y \in C^{2}(I, B)$ is such that $\|y\| \leqslant R$ and $\left\|y^{\prime \prime}\right\| \leqslant \psi\left(\| y^{\prime} \mid\right)$, then $\left\|y^{\prime}\right\| \leqslant M$.

In some of the results which follow, condition (3.1) can be replaced by a weaker integral condition on $\psi$. However, we will use (3.1) throughout since it applies to systems of equations without further restriction.

The next lemma in this section deals with the existence of extremal solutions to a scalar equation related to (1.1), (1.2). Consider the boundary value problem

$$
\begin{aligned}
& -y^{\prime \prime}+f\left(x, y, y^{\prime}\right)=0, \quad x \in(0,1), \\
& g\left(y(0), y^{\prime}(0)\right)=0=h\left(y(1), y^{\prime}(1)\right),
\end{aligned}
$$

where $f:[0,1] \times R \times R \rightarrow R ; g, h: R \times R \rightarrow R$.

A function $\alpha:[0,1] \rightarrow R, \alpha \in C[0,1]$ is called a lower solution of (3.3), (3.4) if for every $\bar{x} \in[0,1]$, there exists a neighbourhood of $\eta(\bar{x})$ of $\bar{x}$ such that

$$
\alpha(x)=\max _{1 \leqslant r \leqslant s} \alpha_{r}(x) \quad(s=s(\bar{x})),
$$

where for each $1 \leqslant r \leqslant s, \alpha_{r} \in C^{2}([0,1] \cap \eta(\bar{x}))$ and satisfies

$$
\begin{gathered}
-\alpha_{r}^{\prime \prime}(x)+f\left(x, \alpha_{r}(x), \alpha_{r}^{\prime}(x)\right) \leqslant 0, \quad x \in(0,1), \\
g\left(\alpha_{r}(0), \alpha_{r}^{\prime}(0)\right) \geqslant 0 \geqslant h\left(\alpha_{r}(1), \alpha_{r}^{\prime}(1)\right) .
\end{gathered}
$$

Similarly, a function $\beta:[0,1] \rightarrow R, \beta \in C[0,1]$ is called an upper solution of (3.1), (3.2) if, for every $\bar{x} \in[0,1]$, there exists a neighbourhood $\eta(\bar{x})$ of $\bar{x}$ 
such that

$$
\beta(x)=\min _{1 \leqslant r \leqslant s} \beta(x) \quad(s=s(\bar{x})),
$$

where for each $1 \leqslant r \leqslant s, \beta_{r} \in C^{2}([0,1] \cap \eta(\bar{x}))$ and satisfies

$$
\begin{gathered}
-\beta_{r}^{\prime \prime}(x)=f\left(x, \beta_{r}(x), \beta_{r}^{\prime}(x)\right) \geqslant 0, \\
g\left(\beta_{r}(0), \beta_{r}^{\prime}(0)\right) \leqslant 0 \leqslant h\left(\beta_{r}(1), \beta_{r}^{\prime}(1)\right) .
\end{gathered}
$$

LEMMA 3.2. Let there exist an upper solution $\beta(x)$ and a lower solution $\alpha(x)$ of (3.3), (3.4) satisfying $\alpha(x) \leqslant \beta(x)$ for $x \in[0,1]$ and let $f$ satisfy a Nagumo condition. Then problem (3.4), (3.5) has a minimal solution $y_{\min }$ and a maximal solution $y_{\max }$ such that for any solution $y(x)$ of $(3.4)$, (3.5) the inequalities $\alpha(x) \leqslant y_{\min }(x) \leqslant y(x) \leqslant y_{\max }(x) \leqslant \beta(x)$.

The above lemma is essentially taken from a recent paper of $K$. Schmitt (see reference [9]). Because of the nonlinearities in the boundary conditions a reference for the proof of this lemma to the result in [9] is a little awkward since there it is proved for linear boundary conditions. However, making use of some recent results on problems with non-linear boundary conditions by Gaines and Mawhin [6], it is not difficult to modify the proof in [9] to include these boundary conditions as well.

Remark 3.3. In the proof of Lemma 3.2, $y_{\min }$ is obtained as the infimum of the set of upper solutions $w(x)$ satisfying $\alpha(x) \leqslant w(x) \leqslant \beta(x)$; and $y_{\max }$ is the supremum of the set of lower solutions $w(x)$ satisfying $\alpha(x) \leqslant w(x) \leqslant \beta(x)$.

4. Some notations and definitions. Denote by $E=E(J)$ the linear space of , real valued sequences indexed by $J \subseteq Z$, where $Z$ is the set of integers. An element $y \in E$ will be denoted $y=\left(y_{j}\right)_{j \in J}$ or simply $y=\left(y_{j}\right)$. Let $\Gamma$ denote the linear span of the set of functionals $\left\{\varphi_{j}: \varphi_{j}: E \rightarrow R, \varphi_{j}(z)=z_{j}\right\}$. The set $\Gamma$ is a total set of functionals on $E$.

A function $f:[0,1] \times E \times E \rightarrow E$ will be called $\Gamma$-continuous if it is weakly continuous in the following sense:

$$
\begin{aligned}
& \text { for each } i \in J \text {, the limits } x \rightarrow x_{0}, y_{j} \rightarrow y_{j}^{0}, z_{j} \rightarrow z_{j}^{0}, \\
& \quad \text { for each } j \in J \text { imply the limit } f_{i}(x, y, z) \rightarrow f_{i}\left(x, y^{0}, z^{0}\right) \text {. }
\end{aligned}
$$

Similarly $g, h: E \times E \rightarrow E$ will be called $\Gamma$-continuous if they are $\Gamma$-continuous considered as functions defined on $[0,1] \times E \times E$.

Let $p$ be a fixed element of $E$ with $p_{j}>0$ for all $j \in J$ and let $E$, denote the Banach space of sequences in $E$ with finite norm

$$
|\boldsymbol{y}|_{p}=\sup \left\{p_{j}\left|y_{j}\right|: j \in J\right\} \text {. }
$$

Let $\Gamma_{p} \subseteq \Gamma$ be the set of linear functionals on $E_{p}$ defined by $\Gamma_{p}=\left\{\varphi_{j} \in \Gamma\right.$ : 
$\left.\varphi_{j}(y)=p_{j} y_{j}, y \in E_{p}, j \in J\right\}$. By $K_{r}$ we denote the cone in $E_{p}$ generated by the linear functionals $\Gamma_{p}$, that is to say, $K_{r}=\left\{y \in E_{p}: \varphi_{j}(y) \geqslant 0, \varphi_{j} \in \Gamma_{n}, j \in J\right\}$. This cone determines the natural order relation on $E_{p}$ :

$$
y \leqslant z \quad \text { iff } \quad y_{i} \leqslant z_{i} \quad \text { for all } i \in J .
$$

Furthermore, int $K_{p}$, the interior of $K_{p}$, is non-empty and we define:

$$
y<z \quad \text { iff } \quad y_{i}+\frac{\delta}{p_{i}} \leqslant z_{i} \quad \text { for all } i \in J \text { and some } \delta>0
$$

in other words, $z-y \in$ int $K_{p}$.

Define the elements $e$ and $e^{j}$ by the equations

$$
e=\left(p_{i}^{-1}\right), \quad e^{i}=\left(\delta_{i j}\right),
$$

where $\delta_{i j}$ is the Kronecker delta. Note that $e \in$ int $K_{p}$ and $|e|_{p}=1$. Given $i \in J$, a point $(x, y, z) \in[0,1] \times E \times E$, and any real number $\gamma$, we define (see reference [14])

$$
f_{i}(x,[y: \gamma], z)=f_{i}\left(x, y+\left(\gamma-y_{i}\right) e^{i}, z\right) .
$$

The functions $g_{i}([y ; \gamma], z)$ and $h_{i}([y ; \gamma], z)$ are defined similarly. The expression $y+\left(\gamma-y_{i}\right) e^{i}$ replaces the $i$-th component of the sequence $y$ with the number $\gamma$. In a similar fashion, if $N_{i} \subseteq J$, we define, for $\gamma \in E\left(N_{i}\right)$,

$$
f_{i}(x,[y ; \gamma], z)=f_{i}\left(x, y+\sum_{j \in N_{i}}\left(y_{i}-y_{i}\right) e^{j}, z\right) .
$$

The expression $y+\sum_{j \in N_{l}}\left(\gamma_{j}-y_{j}\right) e^{i}$ replaces entries from the sequence $y$ whose indices come from the set $N_{i}$ with corresponding entries from the sequence $\gamma$.

We will say that a function $F:[0,1] \times E \times E \rightarrow E$ satisfies a Kanke condition on a set $\Sigma \subseteq[0,1] \times E \times E$ if the following holds:

$$
\begin{aligned}
(x, y, z),(x, \bar{y}, \bar{z}) \in \Sigma, y \leqslant \bar{y}, y_{i}= & \bar{y}_{l}, z_{i}=\bar{z}_{l} \\
& \quad \text { implies } f_{i}(x, y, z) \geqslant f_{i}(x, \bar{y}, \bar{z}) .
\end{aligned}
$$

We will make extensive use of this condition in the results presented in the sections to follow. It is important to note that if $\boldsymbol{F}$ satisfies a Kamke condition on $\Sigma$, then $F_{i}(x, y, z)=F_{i}\left(x, y, z_{i}\right)$ on $\Sigma$, i.e., $F_{i}$ is independent of $z_{j}, j \neq i$. If $\boldsymbol{F}: E \times E \rightarrow E$, then $\boldsymbol{F}$ is said to satisfy a Kamke condition, provided it satisfies (4.2) considered as a function defined on $[0,1] \times E \times E$.

If $p \in E$, the subspace $E_{p}^{0}$ is defined by

$E_{p}^{0}=\left\{u \in E_{p}\right.$ : for every $\varepsilon>0$ there exists a finite subset

$$
\left.N(\varepsilon) \text { of } J \text { such that } p_{f}\left|u_{j}\right|<\varepsilon \text { for all } j \in J \backslash N(\varepsilon)\right\} \text {. }
$$


5. Maximal and minimal solutions for (1.1), (1.2).

THEOREM 5.1. Let there exist functions $\alpha, \beta \in C \Gamma^{2}([0,1], E)$ satisfying $\boldsymbol{\alpha}(x) \leqslant \boldsymbol{\beta}(x)$ and

$$
\begin{array}{ll}
-D^{2} \alpha(x)+f(x, \alpha(x), D \alpha(x)) \leqslant 0, & g(\alpha(0), D \alpha(0)) \geqslant 0 \geqslant h(\alpha(1), D \alpha(1)) \\
-D^{2} \beta(x)+f(x, \beta(x), D \beta(x)) \geqslant 0, & g(\beta(0), D \beta(0)) \leqslant 0 \leqslant h(\beta(1), D \beta(1)) .
\end{array}
$$

Let $f, g$, and $h$ be $\Gamma$-continuous and satisfy a Kamke condition on the set $\Sigma=\{(x, y, z): x \in[0,1], \alpha(x) \leqslant y \leqslant \beta(x), z \in E\}$. Let each $f_{i}$ satisfy a Nagumo condition with Nagumo function $\psi_{i}(s)$ on the set $\Sigma$. Suppose further that $g_{i}(y, z)$ and $h_{i}(y, z)$ are non-decreasing in $z_{i}$ for fixed $y \in E$ and each $i \in J$. Then there exists an increasing sequence of functions $\left\{\alpha^{n}\right\}$ satisfying (5.1) and a decreasing sequence $\left\{\beta^{n}(x)\right\}$ satisfying (5.2) such that $\lim _{n \rightarrow \infty} \alpha^{n}(x)=y_{\min }(x)$ and $\lim \beta^{n}(x)=y_{\max }(x)$.

Proof. Define $L=\left\{w \in C \Gamma^{2}([0,1], E):(x, w(x), D w(x)) \in \Sigma\right.$ and $w$ satisfies (5.1) $\}$ and similarly $U=\left\{w \in C \Gamma^{2}([0,1], E):(x, w(x), D w(x)) \in \Sigma\right.$ and $w$ satisfies (5.2) $\}$. Let $w \in L$ and consider the sequence of scalar problems:

$$
\begin{gathered}
-y^{\prime \prime}+f_{i}\left(x,[w(x) ; y], y^{\prime}\right)=0, \quad x \in(0,1), \\
g_{i}\left([w(0) ; y(0)], y^{\prime}(0)\right)=0=h_{l}\left([w(1) ; y(1)] ; y^{\prime}(1)\right)
\end{gathered}
$$

for $i \in J$. One easily verifies that $\alpha(x)=w_{i}(x)$ is a lower solution $\beta(x)=\beta_{i}(x)$ is an upper solution and that the other hypotheses of Lemma 3.2 are fulfilled as well. Therefore (5.3), (5.4) has a minimal solution which we denote $\hat{y}_{i}(x)$. The equation $S w=\hat{y}$, where $\hat{y}(x)=\left(\hat{y}_{i}(x)\right)$ defines a mapping on $L$. The image $\hat{y}(x)$ of this mapping has the following properties: (i) $w_{i}(x) \leqslant \hat{y}_{i}(x)$ $\leqslant \beta_{i}(x)$; (ii) $\hat{y}_{i}(x)$ satisfies the inequalities

$$
\begin{aligned}
-y_{i}^{\prime \prime}(x)+f_{i}\left(x, \hat{y}(x), \hat{y}_{i}^{\prime}(x)\right) \leqslant-\hat{y}_{i}^{\prime \prime}(x)+f_{i}\left(x,\left[w(x) ; \hat{y}_{i}(x)\right], \hat{y}_{i}(x)\right) & =0, \\
g_{i}\left(\hat{y}(0), \hat{y}_{i}^{\prime}(0)\right) \geqslant g_{i}\left(\left[w(0) ; y_{i}(0)\right], y_{i}^{\prime}(0)\right) & =0, \\
h_{i}\left(\hat{y}(1), \hat{y}_{i}^{\prime}(1)\right) \leqslant h_{i}\left(\left[w(1) ; \hat{y}_{i}(1)\right], \hat{y}_{i}^{\prime}(1)\right) & =0 ;
\end{aligned}
$$

and (iii) if $y(x)$ is any solution of (1.1), (1.2), then $\hat{y}_{t}(x) \leqslant y_{i}(x)$. Of these properties (i) follows from Lemna 3.1, (ii) from the Kamke condition and (iii) from the fact that $y_{1}$ is an upper solution for (5.3), (5.4). together with Remark 3.3. It follows that $S w \in L$ and that $w \leqslant S w$.

Define a sequence $\left\{a^{n}\right\}$ of elements of $L$ by setting

$$
a^{0}=\alpha, \quad \alpha^{n}=S \alpha^{n-1}, \quad n=1,2, \ldots
$$

The properties listed above show that $\alpha^{n}$ is a non-decreasing sequence from $L$ which is bounded above in the partial ordering of $E$ by $\beta(x)$. Let $i$ be a fixed, but otherwise arbitrary, element of $J$ and consider the sequence of 
real valued functions $\left\{\alpha_{i}^{n}(x)\right\}$. The function $\alpha_{i}^{\prime \prime}(x)$ satisfies the differential equation

$$
-\alpha_{i}^{n \prime \prime}(x)+f_{i}\left(x,\left[\alpha^{n-1}(x) ; \alpha_{i}^{n}(x)\right], \alpha_{i}^{n \prime}(x)\right)=0
$$

the boundary conditions

$$
g_{i}\left(\left[\alpha^{n-1}(0) ; \alpha_{i}^{n}(0)\right], \alpha_{i}^{n \prime}(0)\right)=0=h_{i}\left(\left[\alpha^{n-1}(1) ; \alpha_{i}^{n}(1)\right], \alpha_{i}^{n \prime}(1)\right),
$$

and the inequalities

$$
\alpha_{i}(x)=\alpha_{i}^{0}(x) \leqslant \ldots \leqslant \alpha_{i}^{n-1}(x) \leqslant \alpha_{i}^{n}(x) \leqslant \ldots \leqslant \beta_{i}(x) .
$$

Inequalities (5.7) imply that $\left\{\alpha_{i}^{n}\right\}$ converges pointwise to a function $y_{i}(x)$. From (5.5) and the Nagumo condition it follows that the sequence $\left\{\alpha_{i}^{n \prime}(x)\right\}$ is bounded by a constant $M_{i}$ which depends only on $\psi_{i}$ and $\Sigma$. Hence the sequence $\left\{\alpha_{i}^{n}(x)\right\}$ is uniformly convergent to $y_{i}(x)$ and that $y_{i}(x)$ is continuous.

To show that the sequence $\left\{\alpha_{i}^{n \prime}(x)\right\}$ is also relatively compact, it is sufficient, in view of equation (5.5), to show that $f_{i}\left(x, y, z_{i}\right)$ is bounded on the set $\Sigma_{M_{i}}=\left\{(x, y, z) \in \Sigma:\left|z_{j}\right| \leqslant M_{i}, j \in J\right\}$. Suppose to the contrary that this is not the case. Then there is a sequence $\left\{\left(x^{n}, y^{n}, z^{n}\right)\right\}$ in $\Sigma_{M_{i}}$ such that $\left|f_{i}\left(x^{n}, y^{n}, z_{i}^{n}\right)\right| \rightarrow \infty$ as $n \rightarrow \infty$. By the inequalities in the definition of the set $\Sigma_{M_{i}}$ there exists a subsequence $\left\{\left(x^{n_{k}}, y^{n_{k}}, z^{n_{k}}\right)\right\}$ such that $x^{n_{k}} \rightarrow x^{0}, y_{j}^{n_{k}} \rightarrow y_{j}^{0}$, and $z_{j}^{n_{k}} \rightarrow z_{j}^{0}$ for every $j \in J$ as $n_{k} \rightarrow \infty$ and $\left(x^{0}, y^{0}, z^{0}\right) \in \Sigma_{M_{i}}$. But this leads to a contradiction, since by $\Gamma$-continuity then $\left|f_{i}\left(x^{n_{k}}, y^{n_{k}}, z_{i}^{n_{k}}\right)\right|$ $\rightarrow\left|f_{i}\left(x^{0}, y^{0}, z_{i}^{0}\right)\right|<\infty$.

Thus it follows that the sequence $\left\{\alpha_{i}^{n^{\prime}}(x)\right\}$ is uniformly bounded and equicontinuous and hence has a subsequence which converges to $y_{i}^{\prime}(x)$ and that $y_{i}(x)$ is continuous. Because of the monotonicity of the sequence $\left\{\alpha^{n}(x)\right\}$, both subsequences $\left\{\alpha_{i}^{n_{k}}(x)\right\}$ and $\left\{\alpha_{i}^{n_{k^{\prime}}}(x)\right\}$ converge uniformly to $\left\{y_{i}(x)\right\}$. Making use of the $\Gamma$-continuity of $f(x, y, z)$ and the uniform convergence of the sequences $\left\{\alpha_{i}^{n}(x)\right\}$ and $\left\{\alpha_{j}^{n \prime}(x)\right\}$ (where we have renumbered $n=n_{k}$ ) we pass the limit in (5.5) and (5.6) and obtain that $y(x)=\left(y_{i}(x)\right)$ is a solution to (1.1), (1.2). That $y(x)=y_{\min }(x)$ follows from property (iii).

The result concerning the sequence $\left\{\boldsymbol{\beta}^{n}(x)\right\}$ is obtained in a similar manner by defining a mapping $T: U \rightarrow U$ using equations (5.5), (5.6).

Remarks 5.2. (1) If the function $f(x, y, z)$ is row finite, i.e., for each $i$ the function $f_{i}(x, y, z)$ depends on components of $y$ and $z$ coming from finite subsets, say $N_{i}$ and $N_{i}^{\prime}$ of the index set $J$, then $f(x, y, z)$ will be $\Gamma$-continuous if each $f_{i}(x, y, z)$ is continuous as a function on $[0,1] \times R^{n_{i}} \times R^{n_{i}}$, where $n_{i}$ is the number of elements in $N_{i}$ and $n_{i}^{\prime}$, the number in $N_{i}^{\prime}$.

(2) Theorem 5.1 is also true for uncountable index sets $J$ with the one additional restriction, that there exists a positive constant $K_{i}=K_{i}\left(M_{i}\right)$ for 
each $i$ such that

$$
\left|f_{i}\left(x, y, z_{i}\right)\right| \leqslant K_{i}
$$

for all $(x, y, z) \in \Sigma_{M_{i}}$. The argument used in the proof of Theorem 5.1 to show that $f_{i}\left(x, y, z_{i}\right)$ is bounded on $\Sigma_{M_{i}}$ cannot be used if the index set is not countable.

(3) The result in Theorem 5.1 can also be obtained for equation (1.1) together with periodic conditions

$$
y(x+\omega)=y(x)
$$

using some results by Schmitt (see for example reference [9]) and the argument employed in the proof of Theorem 5.1.

6. Monotone approximation in the case of uniqueness of solutions. From a computational point of view, the iteration technique used in Section 5 does not lead to an effective algorithm for approximating solutions to (1.1), (1.2) if $J$ is infinite because each iteration requires the solution of an infinite number of one-dimensional boundary value problems. The approximation of solutions to problems like (1.1), (1.2) requires as a first step a truncation to some type of related finite system. In this section we present another iteration scheme based on truncation for the solution of (1.1), (1.2) which, in the case where (1.1), (1.2) is uniquely solvable, gives a method for local estimation of the truncation error.

The uniqueness portion of the results in this section follow from Theorems 2.1 and 2.2. To satisfy the requirements of the results from Section 2 requires some growth conditions on $f(x, y, z), g(y, z)$ and $h(y, z)$ and on the solutions to the differential inequalities. We will obtain the conditions by formulating the problem in $E_{p}$ for a suitable sequence $p$, rather than in $E$.

The following condition is stronger than the Kamke condition used in Section 5 and is sufficient to guarantee that hypothesis (i) of Theorem 2.1 is satisfied. We will say that $F:[0,1] \times E_{p} \times E_{p} \rightarrow E_{p}$ satisfies condition $(\mathrm{H})$ on a subset $\Sigma \subseteq[0,1] \times E_{p} \times E_{p}$ if

$$
\begin{aligned}
(x, y, z) ;(x, \bar{y}, \bar{z}) \in & \Sigma, \\
& p_{i}\left(y_{i}-\bar{y}_{i}\right)=\inf \left\{p_{j}\left(y_{j}-\bar{y}_{j}\right): j \in J\right\} \leqslant 0, \quad z_{i}=\bar{z}_{i},
\end{aligned}
$$

implies

$$
F_{i}(x, y, z) \leqslant F_{i}(x, \bar{y}, \bar{z}) .
$$

Further, we say that $F_{i}$ satisfies condition $\left(\mathrm{H}^{\prime}\right)$ if strict inequality in (6.1) implies that strict inequality holds in (6.2).

Let $J_{n}$ be a sequence of finite subsets of $J$ with $J_{n} \subseteq J_{n+1}$ and $\lim _{n \rightarrow \infty} J_{n}=J$. Let $w(x)$ be a continuous function $w:[0,1] \rightarrow E_{p}$ and consider the sequence of finite dimensional boundary value problems defined componentwise for $i \in J_{n}$ by 


$$
\begin{gathered}
-y_{i}^{\prime \prime}+f_{i}\left(x,[w(x) ; y], y_{i}^{\prime}\right)=0 \quad x \in(0,1), \\
f_{i}\left([w(0) ; y(0)], y_{i}^{\prime}(0)\right)=0=h_{i}\left([w(1) ; y(1)], y_{i}^{\prime}(1)\right) .
\end{gathered}
$$

THEOREM 6.1. Let there exist $\alpha, \beta \in C\left([0,1], E_{p}^{0}\right) \cap C \Gamma_{p}^{2}\left([0,1], E_{p}\right)$ satisfying $\alpha(x) \leqslant \beta(x)$ and inequalities (5.1), (5.2). Let $f(x, y, z)$ satisfy a Nagumo condition with respect to $z$ with Nagumo function $\psi(s)$ on the set $\Sigma=\left\{(x, y, z) \in[0,1] \times E_{\mathrm{p}} \times E_{\mathrm{p}}: \alpha(x) \leqslant y \leqslant \beta(x),\right\}$ and satisfy a Lipschitz condition on closed, bounded subsets of $\Sigma$ with respect to $z$. Let $f: \Sigma \rightarrow E_{p}$ and $g, h: \Sigma \rightarrow E_{p}$ and be $\Gamma_{p}$-continuous on $\Sigma$.

Let $f(x, y, z)$ satisfy $(\mathrm{H})$ and let $-\boldsymbol{g}(\boldsymbol{y}, \mathrm{z})$ and $\boldsymbol{h}(\boldsymbol{y}, \mathrm{z})$ satisfy $\left(\mathrm{H}^{\prime}\right)$ on the set $\Sigma$ and suppose $g_{i}\left(y, z_{i}\right)$ and $h_{i}\left(y, z_{i}\right)$ are non-decreasing in $z_{i}$ for each $y \in E_{p}$, $i \in Z$. Finally let $f(x, y, z), g(y, z)$ and $h(y, z)$ be semicontinuous with respect to $y$ in the following sense: there exists a continuous function $d: R \rightarrow[0, \infty)$ such that $d(0)=0, d(s)$ is increasing for $s>0, d(-s)=d(s)$ and such that

$$
\begin{array}{r}
f_{i}\left(x, y-\sigma e^{i}, z_{i}\right)-f_{i}\left(x, y, z_{i}\right) \geqslant-d(\sigma), \\
g_{i}\left(y, z_{i}\right)-g_{i}\left(y-\sigma e^{i}, z_{i}\right) \geqslant-d(\sigma), \\
h_{i}\left(y-\sigma e^{i}, z_{i}\right)-h_{i}\left(y, z_{i}\right) \geqslant-d(\sigma) .
\end{array}
$$

Then the sequences $\left\{\boldsymbol{\beta}^{n}\right\}$ and $\left\{\boldsymbol{\alpha}^{n}\right\}$, defined by the equations

$$
\beta^{0}(x)=\beta(x), \quad \beta^{n}(x)=\left[\beta(x) ; y^{n}(x)\right]
$$

and

$$
\alpha^{0}(x)=\alpha(x), \quad \boldsymbol{\alpha}^{n}(x)=\left[\alpha(x) ; \bar{y}^{n}(x)\right],
$$

where $y^{n}(x)$ is the solution of $(6.3-4)_{n}$ with $w(x)=\beta(x)$ and $\bar{y}^{n}(x)$ is the solution of (6.3-4) with $w(x)=\alpha(x)$, converge monotonically from above and below, respectively, to the unique solution $y(x)$ of (1.1), (1.2) in $C\left([0,1], E_{p}\right) \cap C \Gamma_{p}^{2}\left([0,1], E_{p}\right)$.

Proof. Problem (6.3-4) $)_{1}$ satisfies the hypotheses of Theorem 5.1 with index set $J=J_{1}, \boldsymbol{\beta}(x)=\left(\beta_{i}(x)\right)_{i \in J_{1}}$ and $\boldsymbol{\alpha}(x)=\left(\alpha_{i}(x)\right)_{i \in J_{1}}$. Therefore (6.3-4) has solutions $\boldsymbol{y}^{1}(x)$, respectively $\bar{y}^{1}(x)$, and furthermore the functions $\boldsymbol{\beta}^{1}(x)$ and $\boldsymbol{\alpha}^{1}(x)$ satisfy inequalities $(5.1-2)$ and $\boldsymbol{\alpha}(x) \leqslant \boldsymbol{\alpha}^{1}(x) \leqslant \boldsymbol{\beta}^{1}(x) \leqslant \beta(x)$. Problem (6.3-4) $)_{2}$ satisfies the hypotheses of Theorem 5.1 with index set $J=J_{2}, \boldsymbol{\beta}(x)=\left(\beta_{i}^{1}(x)\right)_{i \in J_{2}}$ and $\boldsymbol{\alpha}(x)=\left(\alpha_{i}^{1}(x)\right)_{i \in J_{2}}$. Therefore $(6.3-4)_{2}$ has a solution $\boldsymbol{y}^{2}(x)$, respectively $\bar{y}^{2}(x)$, and furthermore the functions $\beta^{2}(x)$ and $\boldsymbol{\alpha}^{2}(x)$ satisfy inequalities (5.1-2) and $\boldsymbol{\alpha}^{1}(x) \leqslant \boldsymbol{\alpha}^{2}(x) \leqslant \boldsymbol{\beta}^{2}(x) \leqslant \boldsymbol{\beta}^{1}(x)$. Proceeding inductively in this manner we generate sequences $\left\{\boldsymbol{\alpha}^{n}(x)\right\},\left\{\boldsymbol{\beta}^{n}(x)\right\}$ satisfying $(5.1-2)$ and the inequalities $\alpha(x) \leqslant \ldots \leqslant \boldsymbol{\alpha}^{n-1}(x) \leqslant \alpha^{n}(x) \leqslant \ldots$ $\ldots \leqslant \beta^{n}(x) \leqslant \beta^{n+1}(x) \leqslant \ldots \leqslant \beta(x)$.

An argument similar to that used in Theorem 5.1 shows that 
$\lim _{n \rightarrow \infty} \alpha^{n}(x)=y(x)$ and $\lim _{n \rightarrow \infty} \beta^{n}(x)=\bar{y}(x)$ exist and are solutions in $E$. To show that $y, \bar{y} \in C\left([0,1], E_{p}\right) \cap C \Gamma_{p}^{2}\left([0,1], E_{p}\right)$, we note first that the inequalities $\alpha(x) \leqslant y(x) \leqslant \bar{y}(x) \leqslant \beta(x)$ and the fact that $\alpha, \beta \in C\left([0,1], E_{p}^{0}\right)$ imply that $y, \bar{y} \in C\left([0,1], E_{p}^{0}\right) \subseteq C\left([0,1], E_{p}\right)$. It remains to show that $y, \vec{y} \in C \Gamma_{p}^{2}\left([0,1], E_{p}\right)$. It will be sufficient to show that $D y(x)=\left(y_{i}^{\prime}(x)\right)$ and $D \bar{y}(x)=\left(\bar{y}_{i}^{\prime}(x)\right)$ are elements of $E_{\mathrm{p}}$ for each $x \in(0,1)$, since then the hypothesis that $f:[0,1] \times E_{p} \times E_{p} \rightarrow E_{p}$ and equation (1.1) implies $D^{2} y(\dot{x})$ and $D^{2} \bar{y}(x)$ are elements of $E_{p}$. For every $i \in Z$, the function $p_{i} y_{i}$ satisfies the equation

$$
-p_{i} y_{i}^{\prime \prime}+p_{i} f_{i}\left(x, y(x), y_{i}^{\prime}(x)\right)=0
$$

Using the Nagumo function $\psi(s)$ we obtain

$$
\begin{aligned}
p_{i}\left|y_{i}^{\prime \prime}(x)\right| & \leqslant p_{i}\left|f_{i}\left(x, y(x), y_{i}^{\prime}(x)\right)\right| \\
& \leqslant\left|f\left(x, y(x), y_{i}^{\prime}(x) e^{i}\right)-f(x, y(x), 0)\right|_{p}+|f(x, y(x), 0)|_{\boldsymbol{p}} \\
& \leqslant \psi\left(\left|y_{i}^{\prime}(x) e^{i}\right|_{p}\right)+L \\
& \leqslant \psi\left(p_{i}\left|y_{i}^{\prime}(x)\right|\right)+L \equiv \varphi\left(p_{i}\left|y_{i}^{\prime}(x)\right|\right)
\end{aligned}
$$

where $L=\sup \left\{|f(x, y(x), 0)|_{p}: x \in[0,1], \boldsymbol{\alpha}(x) \leqslant \boldsymbol{y}(x) \leqslant \boldsymbol{\beta}(x)\right\}$. The function $\varphi(s)$ satisfies the properties defining a Nagumo function, therefore there exists a constant $M=M(\varphi, \boldsymbol{\alpha}, \boldsymbol{\beta})$ (in particular $M$ is independent of $i$ ) such that $p_{i}\left|y_{i}^{\prime}(x)\right| \leqslant M$. This inequality implies that $|D y|_{p} \leqslant M$, i.e., $D y(x) \in E_{p}$ for each $x \in(0,1)$.

The uniqueness result now follows by applying the result of Theorem 2.2 to $y(x)$ and $\bar{y}(x)$. In Theorem 2.2 we take $B=E_{p}, u_{0}=e, K_{u_{0}}^{*}=\Gamma_{p}$, $w(x)=\bar{y}(x)$ and $v(x)=y(x)$ and conclude $\bar{y}(x) \leqslant y(x)$. Since we have already known that $y(x) \leqslant \bar{y}(x)$ it follows that $y(x)=\bar{y}(x)$.

Remark (1) The iteration scheme used in this section, can also be used in the case where solutions are not unique and in cases where the uniqueness is obtained by means different from the minimum principle used in Theorem 2.1 (see for example [11]). The key restriction in this scheme is that the index set $J$ must be a countable set. In particular the iteration does not depend on the growth conditions on $f$ used in this section.

(2) Since the sequences $\left\{\boldsymbol{\alpha}^{n}(x)\right\}$ and $\left\{\boldsymbol{\beta}^{n}(x)\right\}$ converge monotonically to the same function $y(x)$ their difference $\beta^{n}(x)-a^{n}(x)$ provides an estimate on the difference between the actual solution and either of the approximate solutions $\boldsymbol{\alpha}^{n}(x)$ or $\boldsymbol{\beta}^{n}(x)$. Because of the nature of the iteration scheme used to define $\boldsymbol{\alpha}^{n}(x)$ and $\boldsymbol{\beta}^{n}(x)$ this observation can be used to give local error estimates on the truncation error in approximate solution of (1.1), (1.2). We will illustrate this technique on an application to the method of lines solution to elliptic boundary value problems in the next section. 
7. Approximation of elliptic boundary value problems. In this final section we present an example in the approximation of solutions to elliptic boundary value problems in unbounded domains to which the theorem in Section 6 applies. Let $\Omega=(0,1) \times(0, \infty)$ and consider the following boundary value problem for a perturbation of the Laplace equation:

$$
\begin{gathered}
-\Delta u+g(x, y, u)=0, \quad(x, y) \in \Omega, \\
u(0, y)=\varphi(y), \quad u(1, y)=\psi(y), \quad y \in(0, \infty), \\
u(x, 0)=f(x), \quad x \in[0,1] .
\end{gathered}
$$

Approximate solutions to (7.1), (7.2), (7.3) can be obtained from the solution of the related infinite system of second order ordinary differential equations

$$
\begin{gathered}
-\Delta_{h} u_{i}+g\left(x, . y_{i}, u_{i}\right)=0, \quad x \in(0,1), \quad i \in Z^{+}, \\
u_{i}(0)=\varphi\left(y_{i}\right), \quad u_{i}(1)=\psi\left(y_{i}\right), \quad i \in Z^{+}, \\
u_{0}(x)=f(x), \quad x \in[0,1],
\end{gathered}
$$

where $h>0, y_{i}=i h,-\Delta_{h} u_{i}=-u_{i}^{\prime \prime}-\delta^{2} u_{i}$, and $\delta^{2} u_{i}=h^{-2}\left(u_{i-1}-2 u_{i}+u_{i+1}\right)$, $i \in Z^{+}$. (See reference [11], where this approach is used to investigate existence, uniqueness, and approximation of a problem similar to (7.1), (7.2), (7.3).) The numerical procedure known as the method of lines consists in solving (7.4-6) numerically and using the result as approximate solution to (7.1-3). A discussion of the convergence of this method for non-linear elliptic problems appears in reference [13].

Let $F_{\gamma}$ denote the set of functions

$$
F_{\gamma}=\left\{h(x, y):|h(x, y)| \leqslant C e^{\gamma y}, \gamma \geqslant 0, C>0,(x, y) \in \Omega\right\} .
$$

In order to satisfy the hypotheses of Theorem 6.1, let us assume in (7.1-3) that $\varphi, \psi \in C(0, \infty) \cap F_{\gamma_{0}}$ for some $\gamma_{0}>0$, that $g, g_{u} \in C(\bar{\Omega} \times R)$, $g(x, y, 0) \in F_{\gamma_{0}}$ and $g_{v}(x, y, u) \geqslant \lambda>\gamma_{0}^{2}-\pi^{2}$. Under these conditions it is not difficult to show that for $h>0$ sufficiently small, and $A$ sufficiently large the functions $\boldsymbol{\beta}(x)=\left(\beta_{i}(x)\right)$ and $\boldsymbol{\alpha}(x)=\left(\alpha_{i}(x)\right)$ defined by $\beta_{i}(x)=A \sin \left(\pi_{\mathrm{g}} x\right) \times$ $x \cosh \left(\gamma_{0} y_{i}\right), \pi_{\varepsilon}=\pi /(1+2 \varepsilon), \varepsilon>0$, and $\alpha_{i}(x)=-\beta_{i}(x)$ satisfy the hypotheses of Theorem 6.1 (see reference [10]). Moreover, if we assume that $g(x, y, u) \in F_{\gamma_{1}}$ for $(x, y, u(x, y)) \in F_{\gamma_{0}}$ for some $\gamma_{1}>0$ and that $\lambda>\gamma^{2}>\gamma_{1}^{2}+$ $+\gamma_{0}^{2}-\pi^{2}$ for some $\gamma>0$, then the function $f\left(x, u, u^{\prime}\right)$ defined by the equation

$$
f_{i}\left(x, u, u^{\prime}\right)=-h^{-2}\left(u_{i-1}-2 u_{i}+u_{i+1}\right)+g\left(x, y_{i}, u_{i}\right)
$$

satisfies the hypotheses of Theorem 6.1 with respect to the space $E_{\mathrm{p}}$ for $h>0$ sufficiently small, where $p$ is the element of $E_{p}$ defined by $p_{i}=e^{-\gamma y_{i}}$.

We first note that $\boldsymbol{\alpha}(x)$ and $\boldsymbol{\beta}(x)$ satisfy the hypotheses $\boldsymbol{\alpha}, \boldsymbol{\beta} \in C\left([0,1], E_{p}^{0}\right)$. Moreover, to see that $f$ satisfies $(\mathbf{H})$, let 
$(x, \boldsymbol{u}),(x, \overrightarrow{\boldsymbol{u}}) \in[0,1] \times E_{p}$ be such that $p_{i}\left(u_{f}-\bar{u}_{i}\right)=\inf \left\{p_{j}\left(u_{j}-u_{j}\right): j \in Z^{+}\right\} \leqslant 0$. Then we have

$$
\begin{aligned}
p_{i}\left(f_{i}(x, \vec{u})-f_{i}(x, \boldsymbol{u})\right) & =p_{i}\left(-\delta^{2}\left(\overline{u_{i}}-u_{i}\right)+g\left(x, y_{i}, \overline{u_{i}}\right)-g\left(x, y_{i}, u_{i}\right)\right) \\
& =p_{i}\left[-\delta^{2}\left(\bar{u}_{i}-u_{i}\right)+g_{u}\left(x, y_{i}, u_{i}^{*}\right)\left(\bar{u}_{i}-\dot{u}_{i}\right)\right] \\
& \geqslant p_{i}\left(u_{i}-\overline{u_{i}}\right)\left[h^{-2}\left(\frac{p_{i}}{p_{i}-1}+\frac{p_{i}}{p_{i+1}}-2\right)-\lambda\right]>0
\end{aligned}
$$

for $h>0$ sufficiently small. The last inequality follows since

$$
h^{-2}\left(\frac{p_{i}}{p_{i-1}}+\frac{p_{i}}{p_{i+1}}-2\right)=h^{-2}\left(e^{y h}+e^{-\gamma h}-2\right) \rightarrow \gamma^{2} .
$$

The remaining hypotheses of Theorem 6.1 are easily verified.

To apply the procedure outlined in Section 6 to problem (7.4-6) we fix $h \in\left(0, h_{0}\right)$ so that $\boldsymbol{a}, \boldsymbol{\beta}$, and $f$ fulfil the conditions in Theorem 6.1 and consider the sequence of sets $\left\{J_{n}\right\}$, where $J_{n}=\{1,2, \ldots, n\}$. In this case problem $(6.3-4)_{n}$ consists of $(7.4-6)$ for $j=1, \ldots, n-1$ and, for $i=n,(6.3)$ becomes

$$
-u_{n}^{\prime \prime}-h^{-2}\left(u_{n-1}-2 u_{n}+w_{n+1}(x)\right)+g\left(x, y_{n}, u_{n}\right)=0 .
$$

Let $u^{n}$ denote the solution to (6.3-4) ${ }_{n}$ obtained by setting $w(x)=\alpha(x)$ and $v^{n}$ the solution corresponding to $w(x)=\beta(x)$ and extend these solutions to infinite sequences $\alpha^{n}$ and $\beta^{n}$ be defining $\alpha_{i}^{n}=u_{i}^{n}$ and $\beta_{i}^{n}=v^{n}$ for $i \leqslant n$ and $\alpha_{i}^{n}=\alpha_{i}, \beta_{i}^{n}=\beta_{i}$ for $i>n$. Then from Theorem 6.1 we obtain the following error estimates.

THEOREM 7.1. Let (7.1-3) satisfy the condition listed above. Then for $h>0$ sufficiently small (7.4-6) has a unique solution $\hat{u}$ with $\alpha(x) \leqslant \hat{u}(x) \leqslant \beta(x)$. Moreover, for each $i \in Z^{+}$the inequalities

$$
0 \leqslant \hat{u}_{i}(x)-\alpha_{i}^{n}(x) \leqslant \beta_{i}^{n}(x)-\alpha_{i}^{n}(x) \equiv \varepsilon_{i}^{n}(x)
$$

are satisfied and $\varepsilon_{i}^{n}(x) \rightarrow 0$ monotonically as $n \rightarrow 0$ and this convergence is uniform in $x$ at each fixed $i$.

Remarks (1). In order to simplify presentation of this example we have not included gradient terms in the function $g$. However, since the results in Section 6 permit some $D y$ dependence it is possible to obtain similar results in cases where $g$ does contain gradient terms.

(2) The results presented here also hold for the boundary value problem considered in reference [11].

\section{References}

[1] S. R. Bernfeld and V. Lakshmikantham, Monotone methods for nonlinear boundary value problem in Banach spaces, Nonlinear Analysis 3 (1979), p. 303-316.

[2] J. Chandra, V. Lakshmikantham and S. Leela, $A$ monotone method for infinite systems of nonlinear boundary value problems, Arch. Rat. Mech. Anal, to appear. 
[3] J. Chandra, V, Lakshmikantham and A. R. Mitchell, Existence of solutions of boundary value problems for nonlinear second order systems in a. Banach space, Nonlinear Analysis, 2 (1978), p. 151-168.

[4] K. Deimling, Ordinary differential equations in Banach spaces, Lecture Notes in. Mathematics, No. 596, Springer-Verlag, New York 1977.

[5] N. Dunford and J. T. Schwartz, Linear operators, Part I, Interscience, New York 1957.

[6] R. E. Gaines and J. Mawhin, Ordinary differential equations with nonlinear boundary conditions, J. Diff. Eq. 26 (1977), p. 200-222.

[7] W. Mlak, Note on abstract differential inequalities and Chaplighin method, Ann. Polon. Math. 10 (1961), p. 253-271.

[8] -, and C. Olech, Integration of infinite systems of differential inequalities, ibidem 13 (1963), p. $105-112$.

[9] K. Schmitt, Boundary value problems for quasilinear second order elliptic equations, Nonlinear Analysis 2 (1978), p. 263-304.

[10] -, and R. Thompson, Boundary value problems for infinite systems of second-order differential equations, J. Diff. Eq. 18 (1975), p. 277-295.

[11] -, -, and W. Walter, Existence of solutions of a nonlinear boundary value problem via the method of lines, Nonlinear Analysis 2 (1978), p. 519-535.

[12] R. Thom pson, An invariance property of solutions to second order differential inequalities in ordered Banach spaces, SIAM J. Math. Anal. 8 (1977), p. 592-603.

[13] -, Convergence and error estimates for certain nonlinear elliptic and elliptic-parabolic equations, SIAM J. Numer. Anal. 13 (1976), p. 27-43.

[14] -, Differential inequalities for infinite second order systems and an application to the method of lines, J. Diff. Eq. 17 (1975), p. 421-434.

MATHEMATICS DEPARTMENT, UTAH STATE UNIVERSITY, LOGAN, UTAH

and

DEPARTMENT OF MATHEMATICAL SCIENCES, NORTHERN ILLINOIS UNIVERSITY DEKALB, ILLINOIS

Reçu par la Rédaction le 17. 6. 1978 